\section{Particle Physics Is Well Positioned}

\section{The 1988 ECFA survey of particle physics activities and resources}

The physics potential of the Large Hadron Collider (LHC) being considered by CERN for construction in the existing LEP tunnel (see page 90 for a report on the development of its superconducting magnets) is presently being examined by working groups of the European Committee for Future Accelerators (ECFA). This committee, in representing European particle physics in the CERN Member States, analyses and reports upon resources for research. A final report is expected by the end of the year. In the meantime, ECFA has completed a survey of experimental and theoretical activities and of technical and financial resources, the third in a series dedicated to these topics. The report of the survey (ECFA/RC/90/178: 30 March 1990) provides essential background information on Europe's capacity to launch LHC.

The survey was based upon an almost 100 per cent response to questionnaires distributed in early 1989 to home institutes in the Member States (MS) and to CERN and DESY. It concerned scientists involved in particle physics research or engaged in work whose primary purpose was to examine some property of particles or to test a fundamental principle in particle physics. These people could, in essence, appear as coauthors on a proposal or scientific publication so the ECFA survey adopted a fairly flexible view where some physicists are clearly in a supporting role.

\section{Employment - tenured posts needed?}

As the Table indicates, the number of tenured physicists (those who can stay on until retirement) increased on average by about $4.0 \%$ per annum from 1978 to the date of the survey (1 December 1988). Meanwhile the number of fixed term posts (contracts limited to a few years) increased at about one-half this rate. The difference in growth rates allowed fairly large numbers of fixed term appointees taken on in the late 1970 's for the SPS fixed target experiment and other projects to find permanent employment.

However, by the end of 1988 the ratio of the number of fixed term posts to the total number of (tenured and fixed term) posts was back at the same level as in 1978 (about 0.3 ) owing to a sharp increase in the number of fixed term appointments from mainly eastern Europe for the LEP project at CERN, and for HERA at DESY (the growth rate for 1988 was $16.6 \%$ ).

However, much has changed since 1978. The number of tenured term posts is now in fact more-or-less constant so unless policies are changed over the next few years it will be difficult for the recent influx of fixed term appointees to find permanent posts in the MS and at CERN and DESY.

Graduates - more posts needed?

The data for research students are not so comprehensive. The Table shows that the number working towards a degree requiring them to perform research in particle physics and to write a thesis increased by $6.3 \%$ p.a. from 1978 to 1988 . The ratio of the number of students to the total number of fixed term posts and students had increased from 0.48 to 0.55 over this period.

The report suggested that the growth rate of the number of fixed term posts is now back to the average level for the 1980's (a few percent). The number of students is also growing at its historic rate so it would seem that graduates may have increasing difficulty in finding employment in particle physics. Most institutes commented on a lack of posts (notably for theorists) to keep young graduates and to maintain the staff 1980's.

Technical support - more from home institutes and industry

The total number of senior support staff and technicians in the MS, CERN and DESY contributing to experimental programmes was estimated to have remained the same while the number of support staff per experimental physicist had decreased overall from 1.35 to 0.86 in the period 1978 to 1988 , the ratio had decreased less rapidly in home institutes in the MS (from 1.01 to 0.72 ). This indicates a shift in technical resources to the home institutes which have shown that they were able to make major contributions to experimental programmes in the 1980's.

Maintaining the same overall level of technical support in the MS, CERN and DESY has been compensated for by an increased (but so far unquantified) contribution from industry.

\section{Expenditures - home institutes gain}

The ECFA survey examined expenditures directly related to experimental programmes in particle physics. These did not include salaries and the costs of overheads, technical and administrative support as well as contributions to the running costs of experimental facilities and in some cases, for the construction of accelerators. The rejuvenation that had taken place in the in 1988 as in 1978 (about 2700). However,

overall expenditure in the MS, CERN and DESY had increased by a few per cent from 146 million SFR in 1978 to 189 million SFR in 1988. However, when adjusted for inflation, the annual expenditure per experimental physicist had decreased from $75 \mathrm{kSFR}$ to 61 (1978) kSFR over the period so expenditure had not kept pace with inflation.

This decrease was accompanied by an increasing share of the expenditure being accounted for by more active home institutes. Expenditure per experimental physicist in the home institutes increased slightly from 44 to 52 (1978) kSFR over the survey period. This corresponded to a doubling of the inflation indexed home expenditure in the 14 MS from 77 million SFR in 1978 to 145 million (1978) SFR in 1988 as compared with the CERN contribution which remained constant at about 560 million (1978) SFR throughout the period. In 1988, large provisions for experimental programmes at CERN (notably LEP) were borne by MS directly and not by CERN; in 1978, the SPS fixed target programme came out of the CERN's SPS budget.

\section{CERN contribution}

Funding of home institutes as a percentage of the total contribution to CERN and the institutes still tends to be lower than average for the small MS. In 1988, the combined expenditures at home for the five smallest MS amounted to only $9.6 \%$ of their total contribution as compared with $21 \%$ for the average of all $14 \mathrm{MS}$. The four largest MS in effect favour funding for applied physics.

ECFA concluded by emphasizing that the European particle physics community can look back on a highly successful decade that has brought Europe to the forefront of research at CERN and DESY. Institutes in the Member States have played a vital and increasingly important role in support of accelerator activities, and in non-accelerator physics culminating in new underground laboratories at Frejus and Gran Sasso. Meanwhile, established programmes at CERN and DESY continue to make valuable new contributions. With this record behind it, the community deserves as much support as possible, especially now that it has created a pool of well-trained physicists backed up by strong links with industry.

Tenured staff, fixed term staff and research students working as experimental and theoretical particle physicists in the 14 CERN Member States, CERN and DESY (taken from ECFA Report/RC/90/178)

\begin{tabular}{|l|r|r|r|c|c|}
\hline \multirow{2}{*}{} & \multicolumn{2}{|c|}{ Number } & \multicolumn{3}{c|}{ Growth, \% p.a. } \\
\cline { 2 - 6 } & 1978 & 1988 & $1978-1988$ & 1988 & 1990 \\
\cline { 2 - 6 } Tenured (T) & 1516 & 2260 & 4.0 & -0.4 & $\approx 0$ \\
Fixed term (F) & 741 & 952 & 2.6 & 16.6 & $\approx 0$ \\
Student (S) & 681 & 1149 & 6.3 & - & - \\
$\frac{\mathrm{F}}{\mathrm{F}+\mathrm{T}}$ & 0.33 & 0.30 & & & \\
$\frac{\mathrm{S}}{\mathrm{S}+\mathrm{T}}$ & 0.48 & 0.55 & & & \\
\hline
\end{tabular}

